\title{
A Moment Method Laplace Solver for Low Energy Beam Transport Codes
}

\author{
Christopher K. Allen, Samar K. Guharay, and Martin Reiser \\ Laboratory for Plasma Research \\ University of Maryland, College Park, MD 20742
}

\begin{abstract}
A moment method technique for solving Laplace's equation is presented. The technique is then extended to Poisson's equation to include space charge. The procedure is implemented on a PC and applied to the cases of an electrostatic quadrupole (ESQ) lens and an ellipsoidal bunch in a grounded pipe.
\end{abstract}

\section{INTRODUCTION}

Currently we are engaged in the design and development of a Low Energy Beam Transport (LEBT) section for $\mathrm{H}^{-}$ion beams [1]. We have chosen to employ electrostatic lenses in this design, since for low ion velocities magnetic lenses fail to provide enough focusing for intense beams while gas focusing is intrinsically stochastic. The current prototype consists of $6 \mathrm{ESQ}$ lenses. In order to model the action of such a lens it is neeessary to solve Laplace's equation for the particular lens geometry. Once the lens is characterized electrically, the information may be used in other simulation tools to aid in design.

In general, numerical methods must be utilized to solve Laplace's equation. We present a technique which is fully three dimensional yet is efficient enough for implementation on a $P C$. The efficiency of the technique arises from the fact that it is based on an integral formulation rather than the more common differential form. Instead of solving for electrostatic potential directly, we solve for the surface charge density on conducting bodies. This results in a reduced dimensionality of the problem domain. The integral formulation also readily extends itself to Poisson's equation. Thus, we can model lenses in the presence of charge distributions. Also, since we know the surface charges, we may cvaluate capacitances between various lens elements

\section{NUMERICAL TECHNIQUE}

\section{A. Laplace's Equation}

Letting $\phi$ denote electrostatic potential, the problem is usually seen in the mathematical form

$$
\begin{aligned}
\nabla^{2} \phi(x) & =0 & \forall x \in \Omega, \\
\phi(x) & =f(x) & \forall x \in \Gamma .
\end{aligned}
$$

Here $\Omega$ is the 3D region of interest and $\Gamma$ is its boundary (i.e $\Gamma=\partial \Omega$ ). The function $f$ represents the given boundary values and constitutes the data of the problem. Usually $\Omega$ represents the beam line and $\Gamma$ is the surface of a focusing lens, thus $i$ would be the lens voltage. A finite differencing method would typically attack this problem directly. However, we prefer to work with an integral representation of the problem rather than the differential form [2].

$$
f(x)=\int_{\Gamma} G(x, \xi) \sigma(\xi) d^{2} \xi
$$

where

$$
G(x, \xi)=\frac{1}{4 \pi|x-\xi|}
$$

is the free space Green's function for Poisson's ecpuation The function $\sigma$ is introduced as the new unknown for the problem. It is recognized as the surface charge density on the boundary $\Gamma$. Once $\sigma$ is known, $\phi$ may be recovered via

$$
\phi(x)=\int_{\Gamma} G(x, \xi) \sigma(\xi) d^{2} \xi \quad \forall x \in \Omega .
$$

Note that the dimensionality of the problem has been reduced. In (1), $\phi$ must be solved on $\Omega$, a $3 \mathrm{D}$ subset of $\mathrm{E}^{3}$, while (2) is defined only on the $2 D$ manifold $\Gamma$.

We employ the method of moments to solve (2), the details of which are presented in [3]. Qualitatively, the technique is vary similar to the representation of a guantum mechanical operator in matrix form. We choose a set of expansion functions $\left\{u_{11}\right\}$ on $\Gamma$ which is used to approximate $\sigma$, that is $\sigma(x)=\sum a_{n} u_{n}(x)$, for some set of $a_{n} \in \mathbb{R}$. Another set of functions called weighting (or testing) functions $\left\{\mathrm{v}_{m}^{\prime}\right\}$ is also selected. After expanding (2) in $\left\{u_{n}\right\}$ we take the inner product with each of the $v_{m}{ }^{\prime}$ s. We end up with a series of linear equations where the $a_{n}$ 's are the unknowns. This system may be solved by standard matrix methods. We apply a conjugate gradient algorithm to this end [4]; this is an iterative method which seems to provide fastest convergence.

For our moment method we chose for $\left\{u_{n}\right\}$ a set of piecewise constant functions, constant over the face of a triangle. Specifically, $\Gamma$ is triangulated (approximated by triangles, for example see figure 1) and $\sigma$ is assumed constant 
over each triangle. This selection results in a finite element representation to (2). For the weighting functions we selected Dirac delta functions located at the centroid of each triangle (this is known as pointmatching). This allows fastest evaluation of the inner products and yields good results as long as the triangles are sufficiently regular.

\section{B. Extension to Poisson's Equation}

If we wish to model a charge distribution $\rho$ in the presence of our boundary $\Gamma$, it is convenient to exploit the linearity of the integral operator in (2). That is the potential at the boundary must be the sum of that due to both $\sigma$ and $\rho$.

$$
f(x)=\int_{\Gamma} G(x, \xi) \sigma(\xi) d^{2} \xi+\int_{\Omega} G(x, \xi) \frac{\rho(\xi)}{\epsilon_{0}} d^{3} \xi
$$

or

$$
f(x)-\Phi_{p}(x)=\int_{\Gamma} G(x, \xi) \sigma(\xi) d^{2} \xi
$$

where $\phi_{p}$ is the free space potential due to the charge distribution $\rho$, given by the second integral in (5). Equation (6) is similar in form to (2) and may be solved by applying the method of moments as before to the boundary data $f-\phi_{p}$. Therefore, it is only necessary to determine $\phi_{\rho}$, the free space potential due to $\rho$, in order to apply the method to Poisson's equation. This may be done numerically or analytically (if available).

\section{APPLICATIONS}

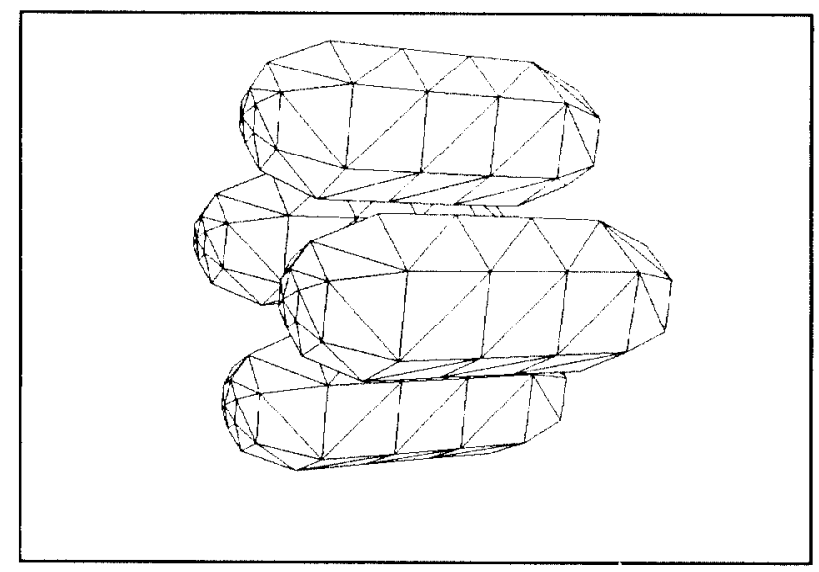

Figure 1: Triangulated ESQ Lens

The preceding technique was implemented implemented with a computer program written in Borland $\mathrm{C}++$ 3.1. The platform was an $\mathrm{i} 486 \mathrm{PC}$ operating at $33 \mathrm{MHz}$ and running Windows 3.1 operating system. All examples where run in double precision arithmetic
A. ESQ Lens

A 3D potential problem is the modeling of an electrostatic quadrupole lens. Figure 1 shows the computer model of an ESQ lens similar to the type used in [1]. It is formed from 4 cigar-shaped electrodes, the beam would enter from the left. Each electrode is $59 \mathrm{~mm}$ long and has a radius: of $12 \mathrm{~mm}$. The aperture of the entire lens is $10.5 \mathrm{~mm}$. Two grounding shunts are located at $\mathrm{z}= \pm 31 \mathrm{~mm}$ (they are not

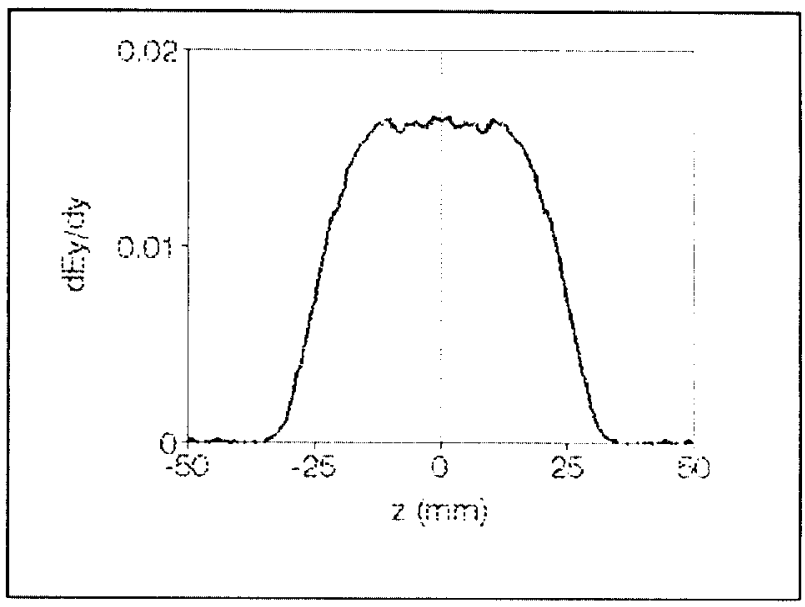

Figure 2: ESQ L.ens Focusing Function

shown in figure 1 to avoid clutter) which provide isolation from adjacent lenses.

The single particle focusing elfect ( the kappa function $\kappa(\mathrm{z})$ ) from such a lenses can he determined from the derivatives $\mathrm{dE}_{\mathrm{x}} / \mathrm{dx}$ and $\mathrm{dE} / \mathrm{dy}$ on axis. Figure 2 shows the computed data for the $y$-plane for the case in which the $x$ plane electrodes are driven to IV and the y-plane electrodes are held at $1 \mathrm{~V}$. The grounding shunt at either end of the lens cause the rapid decay in $\mathrm{dE}_{y} / \mathrm{dy}$

\section{B. Ellipsoidal Bunch in a Pipe}

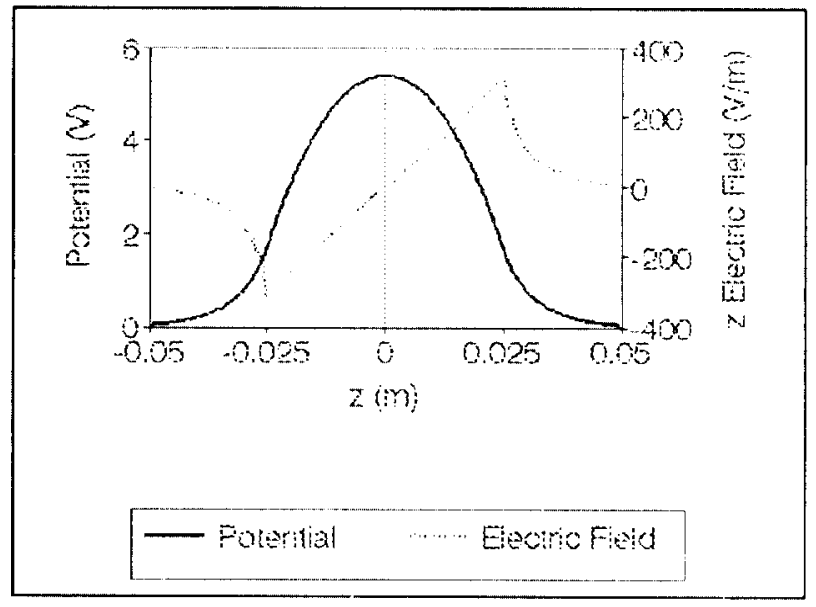

Figure 3: 2.5:1 Ellipsoid in Pipe 
We can use the Poisson extension to simulate a uniform charge density ellipsoid in a conducting cylinder. This situation is useful in modeling cold bunched beams propagating through a beam pipe. There exists an analytic solution for the potential of such an ellipsoid in free space [5]. Thus, it is only necessary to model the pipe (surface charge) numerically.

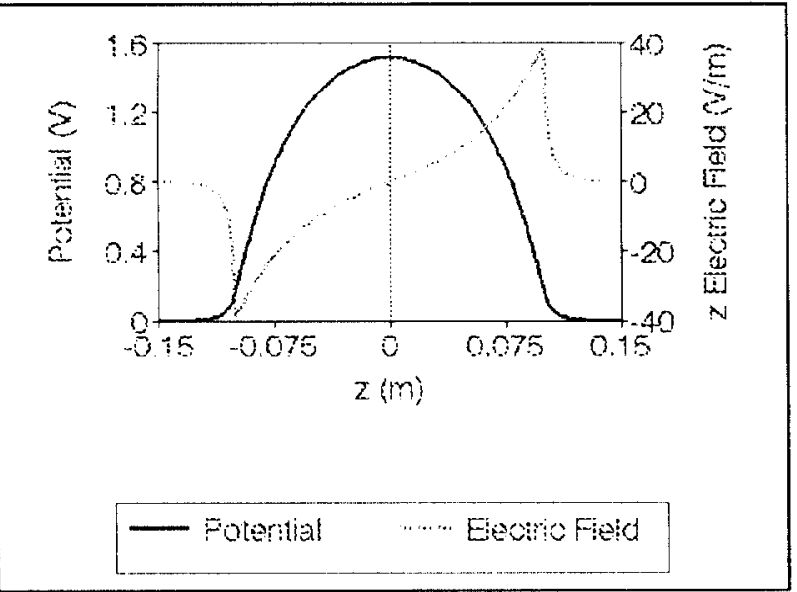

Figure 4: 10:1 Ellipsoid in Pipe

Figure 3 shows the axial potential and $z$ component of electric field for the case of an axissymmetric ellipsoid with major axis $2.5 \mathrm{~cm}$ and minor axis $1 \mathrm{~cm}$ inside a pipe of radius $2 \mathrm{~cm}$. In this case the fields are still relatively linear, the image effects from the pipe are slight. However, in figure 4 we see a very nonlinear field for the case of an ellipsoid with major axis $10 \mathrm{~cm}$ and minor axis $1 \mathrm{~cm}$. We find that after the bunch length becomes comparable to the pipe radius the image effects play an increasing role. The total charge in both cases is $10^{-11} \mathrm{C}$.

The above simulations can be used to determine the so called " $\mathrm{g}$-factor" for bunched beams in cylindrical pipes. A detailed discussion of these results can be found in [6]

\section{CONCLUSION}

The method of moment technique has several advantages and disadvantages. The overall advantage of the technique stems from the fact that only surfaces are considered, rather than $3 \mathrm{D}$ regions. Hence, it is a gond method to model complicated or otherwise arbitrary geometries. For the same system order, we get a higher boundary resolution as compared to finite differencing on a grid. Also, since the surface charges are solved for it is possible to calculate the fields anywhere in space, without interpolation. This fact allows us to apply the method to unbounded situations.
When dealing with the situation of conductors in a vacuum the moment technique is in general quite successful. However, if many dielectrics are present it is probably best to use finite differencing. Also, when it is necessary to know the fields over a large set of points, say when doing many particle simulations, it is probably best to use finite differencing. Computing the potential is a moderatcly expensive process, since we must evaluate (4) at each point, while finite differencing solves for the potential directly.

For the situations discussed the technique is well suited. We need full 3D solutions, yet only for the case of conductors in a vacuum. Also, we are only concerned with the solution data along the beamline axis. Therefore, the number of data points to compute is a minimum.

\section{REFERENCES}

[1] S. K. Guharay, C. K. Allen, M. Reiser, K. Saadatmand,and C. R. Chang, "An ESQ Lens System for Jow Energy Beam Transport Experiments on the SSC Test Stand", (this conference).

[2] I. Stakgold, Green's Functions and Boundary Value Problems (Wiley, NY, 1979) pp. 508-517.

[3] C. K. Allen, S. K. Guharay, and M. Reiser, "Solution of Laplace's Equation by the Method of Moments with Applications to Charged Particle 'Transport", AIP Conf Proc. on Computat. Accelerator Physics, Pleasanton, C $\triangle$ (1993) (to appear).

[4] T. K. Sarkar and E. Arvas, "On a class of Finite Siep Iterative Methods (Conjugate Directions) for the Solution of an Operator Equation Arising in Electromagnetics", IEEL Tram. Antenmas Propagat., vol. AP-33, no. 10, pp. 1058-1066, Oct. 1985.

[5] R. L. Gluckstern, "Scalar Potential for Charge Distributions with Ellipsoidal Symmetry", Fermilab Report TM-1 402 (1986).

[6] M. Reiser, Theory and Design of Charged Parlicle Beams (John Wiley \& Sons, NY, to be published in fall 1993), Ch. 5. 